\title{
L’UOMO E LA VIRILITÀ NELLA NARRATIVA MIGRANTE ITALIANA
}

\author{
Karol KARP \\ Università Niccolò Copernico, Toruń
}

\begin{abstract}
En): The present article deals with the concept of masculinity and the male character in texts characterized as Italian migrant literature. The paper comprises three parts, the first of which focuses on the characters depicted as real men, the second observes those with a tendency to contradict their own masculinity, and the third discusses those that are entirely deprived of it. The reflections on masculinity are set against the social, psychological and sexual backdrops. The analysis features the novels of Lilin, Melliti and Khouma, and a short story by Scego that outlines the various figures of man in migrant literature. In its variety, the story allows for a multidimensional depiction of the problem under examination. The article's theoretical foundation rests on the works of such thinkers as Barthes, Butler, Ferenc, Foucault, Freud, Gilmore, Grosz, Haskell, Mizerkiewicz, Schönborn and Wróbel.
\end{abstract}

Key words (En): Italian migrant literature; men; masculinity; different typologies

Parole chiave (It): letteratura italiana della migrazione; uomo; virilità; tipologie diverse

\section{Introduzione}

È risaputo che fra i rappresentanti della letteratura italiana della migrazione le donne, non di rado desiderose di raccontare le proprie esperienze, ne costituiscono una parte cospicua. Questo fatto può essere visto come una delle ragioni che rende molto visibile, se non addirittura dominante, la presenza femminile nei testi migranti. Tuttavia, non pare però nemmeno corretto affermare che non vi si possano ritrovare figure maschili interessanti, specifiche e complesse. Lo scopo del nostro articolo sarà quello di analizzarne due tipologie completamente diverse l'una dall'altra. Dapprima sarà descritta la figura dell'uomo forte, considerato dagli altri come un esempio da seguire, in seguito le riflessioni saranno dedicate a uomini deboli, che manifestano questa loro caratteristica in varie dimensioni esistenziali. Essi si distinguono per alcuni espliciti atteggiamenti tipicamente femminili e per il fatto che mettono in discussione la propria virilità fino a negarla in modo completo.

La nostra disamina ingloberà i seguenti romanzi: Educazione siberiana (2009) di Nicolai Lilin, Io, venditore di elefanti (1990) di Pap Khouma e Oreste Pivetta, I bambini delle rose (1995) di Mohsen Melliti, e un racconto di Igiaba Scego intitolato Dismatria (2005), scelti per via della ricca varietà di profili maschili ivi contenuti. Vedremo come la virilità si palesa nell'opera di Lilin e quali caratteristiche sono attribuite da Khouma, Scego e Melliti ai loro protagonisti per «de-virilizzarli».

La nozione di virilità sarà qui intesa come un insieme di tratti posseduti da un uomo adulto (GILMORE 1990 : 1) e condizionati socialmente, tradizionalmente e culturalmente (BUTLER 2008). La percezione della virilità dipende completamente da fattori sociali in quanto, come afferma GILMORE (1990 : 9), ogni società compie una distinzione tra maschio e femmina, assegnando agli uomini e alle donne ruoli precisi. Anche l'individuo androgino, continua lo studioso, è costretto a scegliere, molto spesso motivato dai suoi bisogni interiori, una sola identità sessuale per 
risultare agli occhi altrui o una donna o un uomo. Secondo FREUD (1905 : 220), sebbene l'individuo rappresenti una sola identità sessuale, rimane sempre androgino, visto che la femminilità e la virilità non possono essere definite in prospettiva psicologica e biologica. GILMORE (1990 : 11-12) invece caratterizza la virilità contrapponendola alla femminilità: la femminilità è espressa soprattutto dall' attrattività fisica e sessuale, la virilità dalla forza e dalla capacità di confrontarsi con situazioni o nemici pericolosi. Molly HASKELL (1987 : 4) associa la femminilità alla fragilità interiore, alla propensione della donna a lasciarsi guidare dalle emozioni, a provarle in modo intenso. La donna conserva la sua femminilità quando si immerge sovente nel mondo dei sentimenti e svela la sua delicatezza.

\section{Vera virilità}

Con la trama dell'opera di Lilin ${ }^{1}$, contenente una componente autobiografica ${ }^{2}$, ci inoltriamo nella vita della comunità dei siberiani, fortemente maschilista, che già in un primo tempo risulta singolare. I suoi membri sono dei criminali che hanno creato un rigido sistema di regole e di valori trasmessi di generazione in generazione $^{3}$. Quella più giovane è rappresentata da Nicolai, un ragazzo nel ruolo di narratore. Attraverso lo sguardo del protagonista conosciamo le figure di due uomini che godono di un alto status sociale e si configurano come veri e propri eroi.

Lilin ricorre a tre tecniche che non di rado vanno di pari passo: quella della mitizzazione, quella della mitologizzazione e quella della divinizzazione. La mitologizzazione si palesa sotto forma di rimandi intertestuali a una tradizione mitologica precisa, mentre per «mitizzazione» si intende la propensione a creare miti (MIZERKIEWICZ, 2000 : 85-104). Nella tradizione cattolica, la divinizzazione risiede nell'attribuire all'uomo alcuni tratti tipici dell'essere supremo, nonché la capacità di uscire vincitori da situazioni che non lasciano intravedere alcuna speranza. La divinizzazione è «il fine del piano di Dio» (SCHÖNBORN, $2002: 89$ ), può avvenire soltanto attraverso il suo intervento, essa però «non produce un'identità sostanziale con Dio, ma una partecipazione a Dio che deve essere meglio definita» (SCHÖNBORN, 2002 : 90).

Da un lato l'autore del romanzo rende la figura maschile mitica e divina, dall'altro la munisce delle stesse caratteristiche delle grandi autorità antiche che attestano la loro virilità, quali il coraggio, l'ostinazione, l'orgoglio e il senso dell'onore. I suoi protagonisti possono essere identificati con Ercole oppure Achille.

\footnotetext{
${ }^{1}$ Il romanzo è stato un grande successo artistico e commerciale. Il regista Gabriele Salvatores ne ha preparato una versione filmica. Va anche ricordato che ne sono uscite numerose traduzioni in lingue straniere.

2 L'autobiografismo si percepisce ad esempio nel parallelismo tra le origini dell'autore e l'ambientazione della trama. Lilin è nato e cresciuto in Transnistria, località in cui si svolge l'azione.

${ }^{3}$ Vale la pena rilevare la parentela tra l'opera in esame e il romanzo di Roberto Saviano intitolato Gomorra. Viaggio nell'impero economico e nel sogno di dominio della camorra (2006), la quale si estende innanzitutto a livello tematico. Ambedue gli autori tratteggiano un quadro complesso del funzionamento di un' organizzazione criminale particolare, accennano alle relazioni tra i suoi membri, ai modelli di atteggiamento degni di essere seguiti. Un altro elemento importante che accomuna i due testi è la presentazione della condizione dei bambini e degli adolescenti, costretti a crescere in un ambito atipico e a subirne tutte le conseguenze.
} 
Occorre aggiungere che il mito viene qui inteso nell'accezione proposta da Roland BARTHES (1970). Lo studioso lo vede come un elemento importante del discorso diffuso all'interno di una società.

Fra i membri della comunità siberiana campeggia il personaggio di Kruzja, il nonno del narratore. Tutti conoscono la sua storia commovente che suscita ammirazione e rispetto, ma anche compassione e costernazione. Diventato orfano in giovane età, è esposto a varie difficoltà, da quelle economiche a quelle relazionali. Insieme ad altri criminali, si impegna nella lotta contro lo sfruttamento della Siberia da parte dei russi attraverso le rapine dei loro treni carichi di materiali preziosi. Una volta catturato, viene sottoposto a un processo penale e condannato a morte. Il momento dell' esecuzione paradossalmente influirà su tutta la sua vita futura.

Il comandante del plotone ha dato una serie di ordini ai suoi uomini, e quelli hanno preparato i fucili, mirato i loro bersagli e alla fine sparato. Due condannati sono caduti a terra morti, ma il terzo, quello che era in mezzo, continuava a stare in piedi e a guardare la gente. Aveva tutta la camicia sporca di sangue e otto ferite in corpo, ma non cadeva, stava fermo, respirava a fondo l'aria gelida del mattino. Era Kruzja, giovane Urca siberiano (LiLIN, 2009 : 57-58).

Kruzja riesce a sopravvivere all'esecuzione che in teoria avrebbe dovuto ucciderlo, esce vincitore da una situazione che sembrava essere disperata. Per tale motivo viene considerato il simbolo dell'esistenza di Dio, la prova vivente della sua forza, il segno della sua benevolenza e del suo appoggio. La salvezza miracolosa che ha sperimentato lo rende quasi una creatura divina, l'espressione della giustizia che può essere fatta soltanto dall'Essere Supremo. Nell'immaginario dei membri della comunità siberiana incarna ideali dignitosi, nonché la prontezza a sacrificarsi e la resistenza alla sofferenza. La sua storia si propaga in modo veloce, col tempo si rivela come un vero e proprio retaggio mentale trasmesso alle generazioni future. Sebbene Kruzja compiesse azioni illecite, esse servivano per lottare per il bene della terra natale e dei suoi abitanti. In questo modo si presenta come un personaggio onesto, estremamente coraggioso, orgoglioso e più forte della morte. Da vecchio svolge un ruolo importante nel processo di formazione del nipote, modella il suo atteggiamento verso il mondo delle regole sociali in vigore, delle storie affascinanti vissute, nonché delle sue esperienze negative, imbevute di preoccupazioni. $\mathrm{Ne}$ trapela l'immagine di un uomo retto e realizzato, privo di rammarico e rimorsi di coscienza. Nella fase terminale della vita la sua virilità viene espressa dalla saggezza, dalla propensione a formulare giudizi giusti sul comportamento altrui, a dare consigli validi. Sebbene nella cultura la vecchiaia sia generalmente considerata connessa all'ineluttabilità del trapasso, alla mancanza di energia, a una certa rassegnazione, ossia agli elementi che possono essere ritenuti come portatori di devirilizzazione, Lilin presenta Kruzja come un uomo a pieno titolo, sempre un mito vivo, la cui forza non è più fisica, al contrario invece si manifesta attraverso il potenziale intellettuale posseduto e diffuso.

Un'altra figura maschile importante nell'economia del romanzo è il cosiddetto zio Kostič. Per tratteggiarla, lo scrittore migrante fa ricorso alle stesse due tecniche applicate alla costruzione della tipologia di Kruzja, ossia la mitizzazione e la mitologizzazione, tralasciando in modo totale però la divinizzazione. Diversamente dal nonno di Nicolai, Kostič non ha mai partecipato a eventi in cui fosse possibile 
considerarlo l'oggetto di un intervento divino. Il suo personaggio e le vicende di vita sono però un vero e proprio mito. Conformemente alla definizione di Barthes, la mitizzazione del protagonista avviene attraverso la diffusione della sua storia e l'importanza del suo status nella comunità siberiana in questione.

Il vecchio più autorevole in quel locale [...] si chiamava zio Kostič ed era soprannominato « Sčaber». Era un criminale vecchio ed esperto, conosciuto in tutto il Paese; nella nostra comunità e nella mia famiglia parlavano bene di lui, lo trattavano con molto affetto. Era un tipo tranquillo e pacifico, aveva un modo di parlare molto piacevole, si esprimeva con pazienza e umiltà ed era sempre chiaro e diretto (LILIN, 2009 : 141-142).

L'atmosfera mitica che si crea attorno alla figura di Kostič diventa più intensa per via dei tratti del suo carattere. Nell'immaginario sociale risulta un uomo retto, degno di fiducia, ragionevole e modesto. La sua forza fisica, che lo fa assomigliare a Ercole e ad Achille e gli consente di compiere azioni ammirevoli, si palesa esplicitamente, come nell'esistenza di Kruzja, nel periodo della giovinezza. Il narratore rileva la propensione di Kostič a punire chi si comporta in modo controverso e scorretto. Nonostante sia un criminale, a caratterizzarlo è un forte senso di giustizia per così dire umana.

Un giorno Kostič aveva ammazzato di botte una coppia di giovani tossici [...], colpevoli di aver provocato la morte del loro bambino di quattro mesi lasciandolo senza cibo, buttato a morire di fame in un angolo del loro appartamento, tra gli stracci sporchi e i vestiti da lavare. Quella coppia era famosa in città per la sua arroganza (LiLIN, 2009 : 144).

Nei profili maschili tratteggiati da Lilin confluiscono le due varianti del concetto di forza, molto spesso considerate divergenti, ossia la forza fisica, utilizzata nella battaglia, nella lotta, per raggiungere uno scopo preciso, e quella mentale, che sta a esprimere la saggezza. Essere virili significa dunque non solo essere forti e resistenti ai problemi, ma anche intelligenti e giusti. Le esperienze a cui partecipano i protagonisti da giovani li rendono portatori di virilità e permettono loro di acquisire un sapere rilevante affinché possano conservarla nella fase terminale della vita. Lo scrittore migrante non dedica molta attenzione allo studio del loro mondo interiore. Un'analisi profonda dei sentimenti che provano avrebbe di sicuro contribuito alla loro devirilizzazione e ciò non sarebbe in sintonia con il suo scopo, cioè quello di mostrare un uomo completamente privo dei tratti tradizionalmente riservati alle donne.

\section{Virilità contestata}

Il romanzo di Pap Khouma, imbevuto di autobiografismo ${ }^{4}$ e considerato sovente uno dei testi fondatori della letteratura della migrazione (GNISCI, 1998, 2003), ci presenta la figura di un uomo che ha vissuto una dura esperienza migratoria.

\footnotetext{
${ }^{4} \mathrm{La}$ trama racconta le esperienze realmente vissute dall' autore. Esse di sicuro dominano, comunque si mescolano con elementi che sono il frutto di pura finzione letteraria. A livello formale il testo corrisponde dunque alla definizione dell'autofinzione proposta da Serge DoUBRovsKy (1977). Con il termine 'autofinzione' si intende propriamente un testo ibrido in cui confluiscono sia le informazioni che riflettono perfettamente la vita dell' autore sia quelle fittizie.
} 
Diversamente da Lilin, l'autore senegalese studia in modo scrupoloso l'interiorità del suo personaggio principale, fornendo informazioni esplicite sulla sua tipologia. Egli si immerge sovente nel mondo dei sentimenti, e tale atteggiamento, conformemente alle affermazioni di Haskell, è consono alla femminilità.

Nel momento della partenza dal paese d'origine, l'uomo è invaso dal timore per la propria sorte. La visione della nuova realtà che si crea nell'autocoscienza non suscita positività. È fortemente possibile che desideri ritornare in Senegal. Da un lato esprime l'intenzione di trovare migliori condizioni di vita, dall'altro risulta indeciso, debole, perso. Essendo in una situazione di confine, non si distingue per il coraggio che avrebbe mostrato un uomo tipico e così di sicuro mette in dubbio la propria virilità. Tale tendenza lo accompagna nelle fasi successive del suo viaggio verso il nuovo paese. I problemi economici che è costretto a fronteggiare intensificano il suo smarrimento e ne fanno una marionetta pronta a sottomettersi completamente alla volontà altrui. Il protagonista diventa membro di un gruppo di venditori capeggiato da Osman, i cui ordini esegue docilmente. Non è capace di agire indipendentemente, di trovare qualche soluzione per migliorare la propria condizione. Tuttavia, dobbiamo prendere in considerazione che il suo status di immigrato costituisce di sicuro una barriera nello sviluppo individuale. Ciò non significa che non abbia potuto prendere qualche iniziativa e almeno tentare di avere successo. Si nasconde continuamente dalla polizia per evitare la deportazione e prova un'enorme paura. Di fronte ai carabinieri risulta paralizzato e addirittura privo di ragione.

I carabinieri sono due. [...] Non so cosa mi prende. So purtroppo che mi metto a correre come un disperato, con le collane attorno alle braccia, i calzoncini che danzano [...]. Non ho speranze: da una parte c'è il mare, dall'altra l'auto dei carabinieri, alle spalle un carabiniere che mi insegue a piedi, davanti un canale [...]. Mi arrendo. Mi fermo. Il carabiniere mi è addosso, rosso, eccitato, sbuffa e bestemmia. (KHOUMA, 1990 : 99)

Tentando di scappare, il migrante attira l'attenzione dei poliziotti e viene incarcerato per un breve periodo di tempo. La vicenda succitata attesta la sua debolezza e l'ingenuità. Il soggiorno in terra straniera arricchisce l'immagine dell'uomo di nuove caratteristiche, che indubbiamente lo rendono più femminile. Oltre a quelle già rilevate, si devono menzionare anche la sensibilità e la tendenza a essere compassionevole, le quali campeggiano nell'atteggiamento verso le donne senegalesi costrette a prostituirsi in Occidente. Il protagonista esprime senza esitazione il suo disappunto e sottolinea che il fatto gli provoca dolore e delusione, al contempo però non fa niente per aiutarle. Appare come un fantoccio incapace di agire per manifestare quello che considera giusto. Un'altra volta si rivela un personaggio debole, impotente nei confronti della realtà che lo affligge e lo blocca. Un atteggiamento simile riguarda il rapporto che instaura con i locali, visto che la sua provenienza straniera lo espone a vari attacchi dalla loro parte, a umiliazioni e a maltrattamenti. Non è abbastanza forte per lottare in qualche modo, si crede condannato alla sorte che gli è stata riservata, vivendo allo stesso tempo una crisi di personalità e chiudendosi nella propria interiorità. Come dice lui stesso: «Nessuno mi può sentire e l'offesa mi resta dentro, me la trascino appresso per tutta la notte» 
(KHOUMA, 1990 : 65). Inoltre è molto nostalgico, sogna la realtà del paese d'origine, i membri della famiglia, la cui assenza di sicuro intensifica la sua sofferenza. Pare opportuno aggiungere che una tipologia simile di immigrato è delineata nel romanzo Bianco giorno offeso (2004) di Elvira Dones. Ilir risulta molto sensibile, compassionevole verso chi affronta momenti e situazioni difficili, propenso a immergersi nei ricordi sul proprio passato, che di sicuro squilibrano la sua armonia interiore. Gli riaffiorano alla mente le figure dei familiari e altri vari elementi della realtà del paese natale.

Tornando al romanzo di Khouma, si deve mettere in risalto che una contestazione della virilità del protagonista va anche individuata nel suo modo di gestire i soldi guadagnati. Nella prima fase della sua permanenza all'estero, diversamente da molti suoi compagni, l'uomo non risparmia per aiutare i parenti, acquista vestiti e scarpe eleganti. Sebbene disponga di somme modeste, decide di spenderle per prendersi cura dell'aspetto esteriore, desidera essere bello e pieno di fascino. Tale atteggiamento corrisponde alle già rilevate affermazioni di Gilmore, che vede l'attrattività fisica e sessuale come un canale d'espressione della femminilità. La situazione senz'altro si configura in modo paradossale: ci imbattiamo in un migrante povero, quasi privo di mezzi per vivere, impaurito e incapace di riflettere sul proprio futuro. Lo caratterizza la spensieratezza che nella cultura contemporanea viene sovente associata alle giovani donne, alle adolescenti concentrate esclusivamente sulla fisicità, desiderose di intensificare la propria attrattività. L'intenzione di diventare più bello contribuisce alla sua devirilizzazione e non va affatto d'accordo con l'atteggiamento che avrebbe dimostrato un uomo forte e deciso.

\section{Virilità perduta}

Come detto, secondo Freud, ogni creatura umana è androgina. Tale approccio di sicuro rende legittima l'esistenza di disfunzioni e di vari orientamenti sessuali. Nel testo di Igiaba Scego intitolato Dismatria, in cui, come in Khouma e Lilin, sono individuabili alcuni elementi autobiografici ${ }^{5}$, spicca la figura della drag queen Angelique. La scrittrice migrante si focalizza su due aspetti del suo funzionamento: in primo luogo descrive la trasformazione che ha subito, in secondo luogo analizza l'atteggiamento degli altri nei suoi confronti e presenta la sua interiorità.

Prima di diventare una drag queen, l'uomo non tradiva tratti tradizionalmente attribuiti agli omosessuali, «non era lezioso, non era vanesio e non ondeggiava. Camminava ritto su se stesso, in perfetta armonia con il suo peso» (SCEGO, 2005 : 14). Il suo aspetto fisico si poteva dunque definire normale. L'identità sessuale era però completamente atipica e ciò l'ha spinto non solo a travestirsi da donna, ma anche a modificare il proprio corpo in modo che assomigliasse a un corpo femminile. Jerzy Ferenc $(2016: 138)$ mette in evidenza che tale atteggiamento è molto diffuso fra le persone transessuali. Una persona transessuale, dice lo studioso, «desidera vivere, essere accettata e identificata con il sesso opposto».

\footnotetext{
${ }^{5}$ La storia viene raccontata in prima persona da una giovane immigrata, identificabile con la scrittrice.
} 
Angelique ha preso una decisione controversa, ha negato in modo totale la virilità, i valori maschili, si è sottoposta volutamente al processo di devirilizzazione per essere calma nella propria autocoscienza, nonché per informare gli altri su chi è davvero. Il suo corpo ha dunque emesso un messaggio esplicito e andrebbe visto come una mappa da decifrare (GROSZ, 1994, 1995).

È risaputo che per ogni donna è importante essere bella. Ciò è di nuovo consono alla teoria di Gilmore, che lega la femminilità con l'attrattività fisica. La bellezza, come afferma Sylwia SKUZA (2007), riferendosi ai proverbi italiani, può costituire una vera e propria forza che consente di raggiungere certi scopi e di vincere varie battaglie. Angelique forse mirava a diventare bella e attraente, ma intendeva soprattutto svelare la propria sessualità e indubbiamente vi è riuscita. In riferimento alla teoria di Gilmore risulta pertinente ipotizzare che volesse evitare di essere considerata come una creatura androgina, dunque priva di un sesso preciso.

Nei confronti della protagonista gli altri non hanno più dubbi sul suo orientamento sessuale. Il suo aspetto riflette perfettamente i desideri considerati comunemente illeciti che prima custodiva nel proprio animo. Esso può anche costituire una forma di protesta contro le convenzioni socio-culturali. Michel FOUCAULT (2000 : 86-91) rileva che il potere, inteso come rappresentazione di varie istituzioni, influisce sull'identità personale in modo diretto e la forgia. L'identità può liberarsi dal potere solo quando oltrepassa le norme radicate nell' autocoscienza dell'individuo, dall'altro lato l'esistenza del potere genera l'esistenza della protesta. È naturale, quindi, per l'individuo opporsi alle forme portatrici di potere ma nel momento in cui si oppone deve servirsi delle stesse tecniche, degli stessi meccanismi di quelli che lo portano a sottoporsi. Secondo le norme tradizionali, in quanto uomo, Angelique dovrebbe vestirsi in abiti maschili, la protagonista invece si veste da donna. Per palesare la sua identità, si serve dunque di capi di abbigliamento che funzionano come segni evidenti dell'identità femminile. Di conseguenza la sua protesta passa attraverso l'uso di vestiti, ossia degli elementi che prima simboleggiavano il suo imprigionamento.

Il carattere dei rapporti della drag queen con diverse persone si può percepire durante l'incontro familiare nella casa di una sua giovane amica, il cui nome non viene svelato. Angelique si trova al centro dell'attenzione, viene analizzata scrupolosamente dallo sguardo altrui per suscitare sia interesse che perplessità. In genere la si accoglie con entusiasmo, non riesce però a evitare trattamenti ostili.

Mamma mi fissò ancora una volta a lungo. Sorseggiò il caffè rimasto nella tazza. Poi si avvicinò ad Angelique, spiazzandomi. Credevo che si sarebbe rivolta a me, dopo tutto quello scrutare ed esaminare. Invece parlò con la drag e le disse: «Che ci fa lei qui? Non c'entra con noi. Non è della famiglia. Poi signora, non so se lo avrà notato, ma lei è una deviata, omosessuale insomma > (SCEGO, $2005: 17-18$ )

La madre dell'amica di Angelique la attacca per via della sua identità sessuale. Nell'immaginario della donna la rinuncia alla virilità risulta troppo trasgressiva, mette a soqquadro il sistema di valori tradizionali a cui rimane sempre fedele. È giusto anche supporre che la reazione della donna sia la conseguenza dello shock che vive incontrando una persona transessuale probabilmente per la prima volta 
nella sua vita. La transessualità, come rileva Józef WRóBEL (2016 : 114), è rara fra le anomalie sessuali.

La protagonista reagisce alle offese con calma. Si comporta come una persona saggia che non si lascia guidare dalle emozioni e che parla e agisce in modo ragionevole. Questi suoi tratti vengono apprezzati dall'amica: sotto la sua influenza prende la decisione di trasferirsi e di cominciare una nuova vita. Sebbene in un primo tempo Angelique sembri strana, in generale viene presentata come un individuo retto, che non può essere criticato in nessun modo sul piano etico-morale, cosciente del suo status e capace di difendere la propria dignità.

Un altro testo migrante che presenta l'immagine di un uomo che ha perso volutamente la sua virilità è il romanzo di Mohsen Melliti intitolato I bambini delle rose. La trama, ambientata nella Roma contemporanea, si concentra sulla vita dei bambini degli immigrati giunti in Italia. Tra i personaggi del romanzo spicca la figura del signor Enzo, un omosessuale che tradisce la propensione ad abusare dei minorenni. Il suo aspetto esteriore, diversamente da quello di Angelique, non trasmette messaggi espliciti sul suo orientamento, viene svelato soltanto dagli atti sessuali trasgressivi e scioccanti che compie. Il narratore non informa dell' atteggiamento che hanno nei suoi confronti gli altri adulti, accenna soltanto alle pretese delle vittime, tra cui quelle di un ragazzo di nome Nico. Al contrario di Angelique, il signor Enzo risulta privo della capacità di formulare giudizi giusti e di dare consigli. È un uomo completamente devirilizzato che trasgredisce le regole di moralità senza rimorsi di coscienza, mirando a soddisfare i bisogni sessuali totalmente differenti da quelli che nella tradizione sono ritenuti tipici degli uomini veri e propri.

\section{Conclusioni}

Prendendo in considerazione il concetto di virilità, le figure maschili riscontrabili nei testi in esame si possono classificare in tre categorie: quella di individui positivi, quella di individui decisamente negativi e quella di individui «neutrali».

Nel romanzo di Lilin abbiamo a che fare con uomini a pieno titolo che dispongono in modo adeguato non solo della forza fisica ma anche del potenziale intellettuale, aiutano gli altri, riescono a salvarsi miracolosamente dalla morte. Le capacità riflessive fanno sì che in maniera positiva venga anche delineato il quadro dell'uomo che ha negato totalmente la propria mascolinità, trasformandosi fisicamente in donna. Sebbene dalla prospettiva del lettore la trasformazione che ha subito sia senz'altro portatrice di trasgressione, in Scego non è criticata affatto, al contrario, la scrittrice la considera giusta in quanto conforme ai bisogni interiori del soggetto. Il contesto sessuale atipico non rende Angelique un personaggio negativo. In Melliti invece lo stesso contesto fa sì che la figura maschile provochi ripugnanza. Abusando dei ragazzi, il signor Enzo svela la predisposizione all'omosessualità, nega la propria virilità e in un certo senso la propria umanità. La terza categoria individuata, quella «neutrale», riguarda l'uomo migrante, che in alcune situazioni si comporta da donna, si distingue per una grande sensibilità, non ha un carattere forte e dedica un'attenzione molto intensa al proprio aspetto, contestando la virilità, ma 
non rinunciandovi completamente. Il suo atteggiamento di sicuro suscita stupore e perplessità. In fin dei conti può essere ritenuto giustificato, vista la condizione esistenziale particolare del protagonista, lo status di immigrato irregolare, impaurito e desideroso di assaporare una vita migliore.

\section{BIBLIOGRAFIA}

BARTHES Roland (1970), Mit i znak, Warszawa, Państwowy Instytut Wydawniczy. BUTLER Judith (2008), Uwikłani w płeć. Feminizm i polityka tożsamości, Warszawa, Wydawnictwo Krytyki Politycznej.

DONES Elvira (2004), Bianco giorno offeso, Novara, Interlinea.

DouBROVSKY Serge (1977), Fils, Paris, Galilée.

FERENC Jerzy (2016), Operacja korekty płci: czyn legalny czy bezprawny?, in : MORCINIEC Piotr (a cura di), Tożsamość ptciowa i transseksualizm, Opole, Redakcja Wydawnictw Wydziału Teologicznego Uniwersytetu Opolskiego, p. 137-154.

FouCAult Michel (2000), Historia seksualności, Warszawa, Czytelnik.

FREUD Sigmund (1905), Three Essays on the Theory of Sexuality. The Transformations of Puberty, London, Hogarth Press.

GILMORE David (1990), Manhood in the making: cultural concepts of masculinity, New Haven, London, Yale University Press.

GNISCI Armando (2003), Creolizzare l'Europa. Letteratura e migrazione, Roma, Meltemi.

GNISCI Armando (1998), Letteratura italiana della migrazione, Roma, Lilith.

Grosz Elisabeth (1995), Space, Time and Perversion, New York, Routledge.

Grosz Elisabeth (1994), Volatile Bodies: Toward a Corporeal Feminism, Bloomington, Indianapolis, Indiana University Press.

HASKELl Molly (1987), From Reverence to Rape: The Treatment of Women in the movies, Chicago, London, University of Chicago Press.

KHOUMA Pap ; PIVETTA Oreste (1990), Io, venditore di elefanti. Una vita per forza fra Dakar, Parigi e Milano, Milano, Garzanti.

LILIN Nicolai (2009), Educazione siberiana, Torino, Einaudi.

MELliti Mohsen (1995), I bambini delle rose, Roma, Edizioni Lavoro.

MIZERKIEWICZ Tomasz (2000), Mitologizacje. O związkach intertekstualnych z mitologią w powieści polskiej po 1956 r. Pamiętnik literacki, 4, p. 83-104.

SAVIANO Roberto (2006), Gomorra. Viaggio nell'impero economico e nel sogno di domino della camorra, Milano, Mondadori.

SCEGo Igiaba (2005), Dismatria, Roma-Bari, Laterza.

SCHÖNBORn Christoph (2002), Dio inviò suo figlio. Cristologia, Milano, Jaca Book.

SKUZA Sylwia (2007), Bella donna sempre vince - la paremiologia italiana sulla bellezza e sulla bruttezza femminile, in : BRONOWSKI Cezary (a cura di), La figura femminile nella narrativa e nella drammaturgia europea del primo Novecento, Toruń, Wydawnictwo UMK, p. 163-171.

WRÓBEL Józef (2016), Transseksualizm z perspektywy eklezjalnej, in : MORCINIEC Piotr (a cura di), Tożsamość ptciowa i transseksualizm, Opole, Redakcja Wydawnictw Wydziału Teologicznego Uniwersytetu Opolskiego, p. 113-136. 